\title{
A Hybrid Model to Test the Importance of Mechanical Cues Driving Cell Migration in Angiogenesis
}

\author{
A. Stéphanou ${ }^{1 *}$, S. Le Floc'h ${ }^{2}$, A. Chauvière ${ }^{1}$ \\ ${ }^{1}$ UJF-Grenoble 1, CNRS, Laboratory TIMC-IMAG/DyCTiM, UMR 5525, 38041 Grenoble, France \\ ${ }^{2}$ Université de Montpellier II, CNRS, LMGC, UMR 5508, 34095 Montpellier cedex 5, France
}

\begin{abstract}
Many studies are stressing the crucial importance of the mechanical component in angiogenesis, but still, very few models really integrate mechanics. In this paper, we propose to investigate the importance of mechanical cues for cell migration in the context of angiogenesis. We propose a hybrid continuous-discrete model that describes the individual migration of contracting cells on an elastic matrix of fibres. The matrix is described as a continuum whereas the cells are described as discrete elements. We also take into account the degradation of the matrix by proteases. The Young's modulus characterizing the matrix rigidity depends on the local and time-dependent density of matrix fibres. Our results show that acting on the mechanics, specifically on the cell traction force intensity and on the matrix rigidity, can significantly alter cell migration and angiogenesis. First, there is a limited range of traction force intensities for which a vascular network can be obtained. Second, the matrix rigidity plays a role, but only in a very specific range, compatible with the underlying biological process. Alteration of the matrix due to cell degradation appears too small to induce significant changes in cell migration trajectories.
\end{abstract}

Keywords and phrases: angiogenesis, cell migration, cell traction force, matrix degradation, mechanobiology

Mathematics Subject Classification: 35Q92, 65M06, 92C10, 92C15, 92C17

\section{Introduction}

Vascular development has been extensively studied both in vitro and in silico for many years. It is relatively simple to seed endothelial cells on a biogel and observe their spontaneous organization into a network of tubes of varying length depending on factors such as the biogel rigidity [38]. This provides a challenging problem for theoretical investigation, motivated by the fact that the formation of blood vessels is crucial to provide and maintain the adequate flux of resources and waste drainage to sustain the viability of the tissues and normal functioning of the organs.

Two mechanisms of vascular development can be distinguished, vasculogenesis and angiogenesis. Vasculogenesis concerns the differentiation of progenitor cells, the angioblasts, that interact with the

\footnotetext{
${ }^{*}$ Corresponding author. E-mail: angelique.stephanou@imag.fr
} 
surrounding extracellular matrix (ECM) to form new blood vessels. This process mainly occurs in the developing embryo. Angiogenesis, in the other hand, is the formation of new vessels from existing ones through sprouting, branching and anastomosis. The sprouting and branching processes are promoted by growth factors diffusing in the tissue. This explains why theoretical models of angiogenesis, and more specifically tumour-induced angiogenesis, were for many years almost exclusively based on the integration of biochemical cues $[2,8,31]$. At the opposite, vasculogenesis models, where the cell-matrix interaction dominates over the involvement of growth factors, were mainly focused on the integration of mechanical aspects $[23,26,27]$. Those models describe the extracellular matrix as a viscoelastic medium deformed by the contraction force of endothelial cells. The matrix deformations are the cues that drive the organisation of the cells forming the resulting vascular network. A thorough review of the mathematical models for these two processes can be found in [35].

It soon became clear that taking into account cell-matrix interactions in tumour-induced angiogenesis model was crucial to fully address the understanding of this complex process. The cell-matrix interaction is usually minimally taken into account by considering haptotaxis, which is the migration of the cells along gradients of matrix proteins. The gradients appear when cells degrade the matrix. Almost all models now integrate cell-matrix interaction, beyond haptotaxis, in one way or another. This is done, either by looking at the impact of the local topology (fibre orientation) and composition of the ECM using heterogeneous (in density) or anisotropic (in orientation) matrices [1,12,25], or by considering mechanical aspects such as matrix stiffness [33] or cell traction [11,41].

The increase of information on angiogenesis acquired from more and more sophisticated experiments and imaging techniques has promoted and stimulated the development of many new computational hybrid approaches in order to integrate those heterogeneous and multiscale information. For example hybrid models allows one to switch from an explicit description of the matrix fibres to a field description at a higher scale which retains the main characteristics of density and orientation [25]. Cell-centred computational models, based on cellular Potts models, were proposed to investigate the importance of cell elongation in in vitro vasculogenesis [24], to provide a better description of the sprouting and branching processes at the cell scale [10], to account for cell competition for space inducing resistance on migration and proliferation [3], to integrate biochemical events and intracellular signalization [34] to get insights into blocking therapies. The formation of functional blood vessels, corresponding to the explicit description of the blood vessels heterogeneous geometries could also be modelled with a hybrid approach based on a convected element method [30]. Edgar et al. [12] used a hybrid model to account for the branching and elongation processes observed from in vitro angiogenesis experiments. Das et al. [8] proposed a hybrid stochastic-deterministic model that integrates cell state changes from proliferating to apoptotic and intracellular reactions to account for 3D experimental sprout morphologies. Spill et al. [37] proposed a model with a mesoscopic level of description that allows to evaluate the deterministic limit corresponding to stochastic events of proliferation and cell movements. This gives a mean to compare different models and to identify the most pertinent mode of description stochastic-discrete or deterministic-continuous.

Although many studies have stressed the importance of the mechanical component [12, 20,32], still very few models, even among the most recent hybrid ones, takes it explicitly into account. In this paper, we propose to investigate the importance of mechanical cues associated to the molecular cues involved in angiogenesis, focusing specifically on tumour-induced angiogenesis. We note that this was reciprocally done for vasculogenesis, with the incorporation of chemotaxis in a mechanical model [40]. Specifically, we propose a hybrid continuous-discrete model that describes the individual migration of contracting cells on an elastic matrix of fibres. Our model is primarily inspired from [16], where the authors proposed a continuous model of tumour angiogenesis incorporating for the first time cellular traction and viscoelastic effects. This model was then further developed in [4]. Our new model proposes substantial differences with regard to these two models. First, cells and 
ECM are not considered as a contractile viscoelastic continuum. Instead, we consider a homogeneous density of fibres conveying an elastic property to the matrix, where individual cells apply local traction forces. The matrix is therefore described as a continuum but the cells are described as discrete elements. Second, matrix degradation and remodelling are known to be important factors in vascular morphogenesis [9], so we also take into account the degradation of the matrix by proteases. This means that the Young's modulus characterizing the ECM, depends on the local and time-dependent fibre density.

The paper is organized as follows; we first present the computational model detailing the integration of the molecular and the mechanical components. We intentionally separated the continuous formulation of the model from the discrete one, since the discrete part essentially concerns technical calculations. The continuous part thus presents in full the biology and biomechanics of the model. We then present simulation results with two parts. In the first part, we simulate angiogenesis for a range of cell traction force intensities. In the second part, we investigate more closely the contribution of the different mechanical parameters, specifically the impact of the matrix rigidity on the characteristics of cell migration. Finally we discuss the limitations of the model and propose some new directions that would need to be explored.

\section{The computational model}

\subsection{Cell migration}

Tumour angiogenesis is usually modelled on the basis of three terms: a diffusion term to account for the random component of the displacement of endothelial cells, a chemotactic term to account for the influence of growth factors that are mainly responsible for leading vessels growth towards the tumour source and a haptotactic term to account for the cell responsiveness to the density of matrix fibres [2]. In such a model the cell-matrix interaction only relies on this last term.

In our model, we keep these three terms and further assume that the random cell displacement (i.e. diffusion) is biased by some strains generated by the cell traction on the matrix fibres. We assume that only cells located at the tip of the sprouts exert traction forces on the matrix $[13,15]$. The diffusion coefficient, instead of being a constant, is a two-dimensional tensor $\boldsymbol{D}(\varepsilon)$ which reflects its dependency with the local strain tensor $\varepsilon=\frac{1}{2}\left(\nabla \mathbf{u}+\nabla \mathbf{u}^{\mathbf{T}}\right)$, where $\mathbf{u}$ is the displacement vector of the matrix fibres. Specifically, the matrix compression in one direction and its expansion in the perpendicular direction are assumed to modify the matrix fibres alignment and then to influence the direction and velocity of the cells displacements. We use the diffusion tensor initially proposed by [26] and extensively used to describe in vitro angiogenesis $[16,23,27]$. It is given by:

$$
\boldsymbol{D}(\varepsilon)=D_{0}\left(\begin{array}{cc}
2+\varepsilon_{x x}-\varepsilon_{y y} & \varepsilon_{x y}+\varepsilon_{y x} \\
\varepsilon_{x y}+\varepsilon_{y x} & 2-\varepsilon_{x x}+\varepsilon_{y y}
\end{array}\right)
$$

where $\varepsilon_{x x}, \varepsilon_{y y}, \varepsilon_{x y}, \varepsilon_{y x}$ are the components of the strain tensor expressed in two dimensions and $D_{0}$ is the diffusion coefficient accounting for the random migration of the cells in the absence of strain (multiplied by 2).

The continuous equation expressing the combined strain-biased, chemotactic and haptotactic migration of the endothelial cells, with density $n(x, y, t)$, in the simulation domain is thus given by:

$$
\frac{\partial n}{\partial t}=\underbrace{\nabla \cdot[\nabla \cdot(\boldsymbol{D}(\varepsilon) n)]}_{\text {strain-biased diffusion }}-\underbrace{\nabla \cdot(\chi(c) n \nabla c)}_{\text {chemotaxis }}-\underbrace{\rho \nabla \cdot(n \nabla f)}_{\text {haptotaxis }}
$$

where $c(x, y, t)$ and $f(x, y, t)$ represent the concentrations of growth factors and matrix fibres respectively. The function $\chi(c)=\frac{\chi}{1+\alpha c}$ describes the cells loss of sensitivity (tuned with the parameters $\chi$ and $\alpha$ ) as 
the growth factors concentration increases and $\rho$ is a parameter describing the cell sensitivity to the matrix fibres which is assumed to be constant. These terms are kept as they were defined in [39].

\subsection{Molecular component}

The molecular actors involved in angiogenesis are extremely numerous [29]. However we can describe the main features of this complex process by only taking into account three types of proteins: the growth factors $(c)$ which stimulate endothelial cells migration and proliferation; the matrix fibres $(f)$ for which the cells are very sensitive to the density; the matrix degrading enzymes $(m)$ produced by the cells in order to be able migrate through the fibres. For each of these three families of proteins, there exist many different types that is why we are not more specific about them. Governing equations for these proteins are:

$$
\begin{aligned}
& \text { Growth Factors } \frac{\partial c}{\partial t}=-\eta n_{i, j} c+D_{c} \nabla^{2} c \\
& \text { Matrix fibres } \quad \frac{\partial f}{\partial t}=-\gamma m f \\
& \text { Degrading enzymes } \quad \frac{\partial m}{\partial t}=\beta n_{i, j}-\delta m+D_{m} \nabla^{2} m
\end{aligned}
$$

The parameters $\eta, \beta, \gamma, \delta, D_{c}, D_{m}$, designates respectively the consumption rate of growth factors and the production rate of matrix degrading enzymes by the cells, the degradation rate of matrix fibres by the enzymes, the enzyme decay rate and diffusion coefficients of the growth factors and enzymes. $n_{i, j}$ designates the discrete endothelial cells. This notation is used to indicate that this term does not designate a density of endothelial cells but individual cells that we locate on the $(i, j)$ simulation grid. We therefore assume that the this single cell stands for the tip cell of the sprout. We also implicitly assume that stalk cells, located behind the tip cell, proliferate to allow the vessel to grow. All details on the continuous to discrete transition can be found in [2].

\subsection{Mechanical component}

\subsubsection{Cell traction force}

The cells exert traction forces on the matrix as they migrate. To move forward, cells attach to the matrix fibres through specific transmembrane proteins such as integrins. These integrins transmit to the matrix the forces generated by the contraction of the intracellular actomyosin fibres, thus pulling the cell forward. In angiogenesis, the migrating cells are essentially located at the tip of the growing blood vessels which correspond to the sprouts. The tip cells migrate to progress through the tissue and strongly interact with the matrix by forming adhesions to probe the environment and to move in an appropriate direction by producing a traction force [13]. The stalk cells mainly proliferate to form the lumen and the level of interaction with the matrix is less important since these cells preferentially bind together (rather than to the matrix) and are mainly pulled forward by the tip cell [15]. The traction force on the matrix is thus reduced at the level of the stalk cells. We therefore consider in our model that traction forces are exerted locally by one single cell or by a small number of cells, those forming the sprout.

We assume, to make it simple, that only the tip of the sprout is contracting. Each cell generates a dipole-like contractile force $\boldsymbol{F}$ on the matrix oriented in the direction of the sprout growth (Fig. 1). We further assume that the intensity of this force, that we define with the parameter $\tau$, is constant.

In order to describe the mechanical forces distributed throughout the space as the vessels grow, it is required to locate each individual sprout in the domain. This can be done by discretizing the equation (2.2) using the scheme originally proposed in [2], so as to transform this equation into a probabilistic equation 


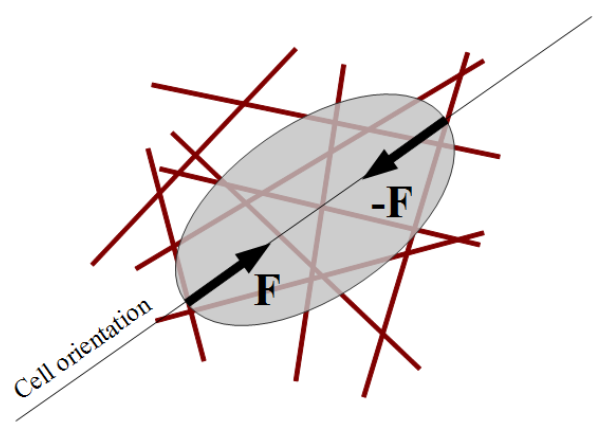

FiguRE 1. The cell traction is represented by a dipole-like force oriented along the cell main axis which corresponds to the direction of migration. The rods represents the matrix fibres, although we do not describe them explicitely in this model.

for individual cell migration. The direction for migration is then determined by a set of probabilities derived from the original continuous equation and integrating the contributions of the different terms.

\subsubsection{Mechanical equilibrium}

In most existing model for vasculogenesis (or in vitro angiogenesis), cell tractions are considered as internal forces of a cell-matrix viscoelastic continuum $[16,23,26,27]$. In the model that we propose, we consider cell tractions as external forces applied on the matrix since only few cells, those located in the sprouts, generate such forces. Due to the small amount of contributing cells and to their sparse distribution on the matrix, matrix and cells cannot be treated as a continuum any more. Therefore, the mechanical equilibrium of the forces applied on the matrix implies that the internal elastic force, due the matrix fibres, balances the two external forces which are the cell traction on the matrix, where $\boldsymbol{e}$ is a vector for the force direction, and a body force reflecting the attachment of the fibres to the rest of the tissue. This force is modulated by the attachment parameter $s$ and depends on the matrix displacement $\boldsymbol{u}[27]$. The force balance equation is given by the divergence of the stress tensor $\boldsymbol{\sigma}$ accounting for the internal forces in the matrix equals to the total external forces (prescribing zero-flux conditions on the domain boundary), that is:

$$
\nabla \cdot \boldsymbol{\sigma}=\tau n_{i, j} \boldsymbol{e}+\frac{s \boldsymbol{u}}{f}
$$

The extracellular matrix is usually described as a viscoelastic material. The elastic property is attributed to the fibres which have the ability to resist the pulling forces of the cells by exerting an elastic restoring force, whereas the viscous property is attributed to the liquid component of the matrix. In this model, we chose to ignore the viscous component to consider the matrix as a pure elastic material. We will restore the viscous component in a further improved model. The elastic stress tensor is thus given by:

$$
\boldsymbol{\sigma}=\frac{E(f)}{1+\nu}\left(\varepsilon+\frac{\nu}{1-2 \nu} \theta \boldsymbol{I}\right)
$$

where $\theta=\nabla \cdot \boldsymbol{u}$ is the dilation, $\nu$ is the Poisson's ratio and $E(f)$ is the Young's modulus. As the cells degrade the fibres, the rigidity of the matrix is altered (noting that the fibres degradation is never total in our simulations). We reflect this fact with a linear dependency of the Young's modulus with the concentration of fibres in the matrix $E(f)=\kappa f$. 


\subsubsection{Matrix displacements}

Development of equation (2.6) gives:

$$
\begin{gathered}
\frac{\partial \sigma_{x x}}{\partial x}+\frac{\partial \sigma_{x y}}{\partial y}=\frac{s u}{f}+\tau n_{i, j} e_{x} \\
\frac{\partial \sigma_{x y}}{\partial x}+\frac{\partial \sigma_{y y}}{\partial y}=\frac{s v}{f}+\tau n_{i, j} e_{y}
\end{gathered}
$$

where $u$ and $v$ are respectively the horizontal and vertical components of the matrix displacement and $e_{x}$ and $e_{y}$ designate the orientation of the $n_{i, j}$ cells for each equation, that is along the $x$-axis and $y$-axis respectively. According to eq. (2.7), we have:

$$
\begin{aligned}
\sigma_{x x} & =\tilde{E}_{f}\left[\varepsilon_{x x}+\tilde{\nu}\left(\varepsilon_{x x}+\varepsilon_{y y}\right)\right] \\
\sigma_{x y} & =\tilde{E}_{f} \varepsilon_{x y} \\
\sigma_{y y} & =\tilde{E}_{f}\left[\varepsilon_{y y}+\tilde{\nu}\left(\varepsilon_{x x}+\varepsilon_{y y}\right)\right] \\
\sigma_{y x} & =\tilde{E}_{f} \varepsilon_{y x}
\end{aligned}
$$

where we have set:

$$
\tilde{E}_{f}=\frac{E(f)}{1+\nu} \quad \text { and } \quad \tilde{\nu}=\frac{\nu}{1-2 \nu}
$$

From now on, we further drop the tildes for clarity.

Since,

$$
\varepsilon=\left(\begin{array}{ll}
\varepsilon_{x x} & \varepsilon_{x y} \\
\varepsilon_{y x} & \varepsilon_{y y}
\end{array}\right)=\left(\begin{array}{cc}
\frac{\partial u}{\partial x} & \frac{1}{2}\left(\frac{\partial u}{\partial y}+\frac{\partial v}{\partial x}\right) \\
\frac{1}{2}\left(\frac{\partial u}{\partial y}+\frac{\partial v}{\partial x}\right) & \frac{\partial v}{\partial y}
\end{array}\right)
$$

the expressions used to calculate the horizontal and vertical displacements of the extracellular matrix can now be derived.

Equation for the displacement $u$

From eq. (2.8), we have:

$$
\begin{gathered}
\frac{\partial}{\partial x}\left(E_{f}(1+\nu) \varepsilon_{x x}+E_{f} \nu \varepsilon_{y y}\right)+\frac{\partial}{\partial y}\left(E_{f} \varepsilon_{x y}\right)=\frac{s u}{f}+\tau n_{i, j} e_{x} \\
E_{f}(1+\nu) \frac{\partial \varepsilon_{x x}}{\partial x}+(1+\nu) \frac{\partial E_{f}}{\partial f} \frac{\partial f}{\partial x} \varepsilon_{x x}+E_{f} \nu \frac{\partial \varepsilon_{y y}}{\partial x}+\nu \frac{\partial E_{f}}{\partial f} \frac{\partial f}{\partial x} \varepsilon_{y y}+E_{f} \frac{\partial \varepsilon_{x y}}{\partial y}+\frac{\partial E_{f}}{\partial f} \frac{\partial f}{\partial y} \varepsilon_{x y}=\frac{s u}{f}+\tau n_{i, j} e_{x} \\
E_{f}(1+\nu) \frac{\partial^{2} u}{\partial x^{2}}+(1+\nu) \frac{\partial E_{f}}{\partial f} \frac{\partial f}{\partial x} \frac{\partial u}{\partial x}+E_{f} \nu \frac{\partial^{2} v}{\partial x \partial y}+\nu \frac{\partial E_{f}}{\partial f} \frac{\partial f}{\partial x} \frac{\partial v}{\partial y}+\frac{E_{f}}{2}\left(\frac{\partial^{2} u}{\partial y^{2}}+\frac{\partial^{2} v}{\partial y \partial x}\right) \\
+\frac{1}{2} \frac{\partial E_{f}}{\partial f} \frac{\partial f}{\partial y}\left(\frac{\partial u}{\partial y}+\frac{\partial v}{\partial x}\right)=\frac{s u}{f}+\tau n_{i, j} e_{x}
\end{gathered}
$$

Equation for the displacement $v$

From eq. (2.9), we have:

$$
\begin{gathered}
\frac{\partial}{\partial x}\left(E_{f} \varepsilon_{x y}\right)+\frac{\partial}{\partial y}\left(E_{f}(1+\nu) \varepsilon_{y y}+E_{f} \nu \varepsilon_{x x}\right)=\frac{s v}{f}+\tau n_{i, j} e_{y} \\
E_{f}(1+\nu) \frac{\partial \varepsilon_{y y}}{\partial y}+(1+\nu) \frac{\partial E_{f}}{\partial f} \frac{\partial f}{\partial y} \varepsilon_{y y}+E_{f} \nu \frac{\partial \varepsilon_{x x}}{\partial y}+\nu \frac{\partial E_{f}}{\partial f} \frac{\partial f}{\partial y} \varepsilon_{x x}+E_{f} \frac{\partial \varepsilon_{x y}}{\partial x}+\frac{\partial E_{f}}{\partial f} \frac{\partial f}{\partial x} \varepsilon_{x y}=\frac{s v}{f}+\tau n_{i, j} e_{y}
\end{gathered}
$$




$$
\begin{gathered}
E_{f}(1+\nu) \frac{\partial^{2} v}{\partial y^{2}}+(1+\nu) \frac{\partial E_{f}}{\partial f} \frac{\partial f}{\partial y} \frac{\partial v}{\partial y}+E_{f} \nu \frac{\partial^{2} u}{\partial x \partial y}+\nu \frac{\partial E_{f}}{\partial f} \frac{\partial f}{\partial y} \frac{\partial u}{\partial x}+\frac{E_{f}}{2}\left(\frac{\partial^{2} u}{\partial x \partial y}+\frac{\partial^{2} v}{\partial x^{2}}\right) \\
+\frac{1}{2} \frac{\partial E_{f}}{\partial f} \frac{\partial f}{\partial x}\left(\frac{\partial u}{\partial y}+\frac{\partial v}{\partial x}\right)=\frac{s v}{f}+\tau n_{i, j} e_{y}
\end{gathered}
$$

The developed elliptic equations (2.15) and (2.16) are then solved using a Successive Over Relaxation (SOR) method with zero-flux conditions at the boundaries [43] (see appendix A for details). We first solve $u(x, y)$ and $v(x, y)$ in the $x$-direction on the first half of the simulation time step $(t \longrightarrow t+\Delta t / 2)$ and in the $y$-direction on the second half of the timestep $(t+\Delta t / 2 \longrightarrow t+\Delta t)$. In order to locate individually each of the sprouts, we track down the displacements of the cells using the discrete stochastic model for cell migration.

\subsection{Discrete stochastic model for individual cell migration}

\subsubsection{Discretization of the general equation}

The displacement of each individual cell, located at the tip of each growing sprout, is obtained from the discretized form of equation (2.2) whose general expression is equivalent to:

$$
\frac{\partial n}{\partial t}=A \frac{\partial^{2} n}{\partial x^{2}}+B \frac{\partial^{2} n}{\partial y^{2}}+C \frac{\partial^{2} n}{\partial x \partial y}+D \frac{\partial n}{\partial x}+E \frac{\partial n}{\partial y}+F n
$$

where $A, B, C, D, E$, and $F$ are coefficients that we need to determine. Discretization of equation (2.17), using a centred Euler finite difference approximation for space and a forward scheme for time, gives:

$$
\begin{aligned}
\frac{n_{i, j}^{t+\Delta t}-n_{i, j}^{t}}{\Delta t} & =A \frac{n_{i+1, j}^{t}-2 n_{i, j}^{t}+n_{i-1, j}^{t}}{(\Delta x)^{2}} \\
& +B \frac{n_{i, j+1}^{t}-2 n_{i, j}^{t}+n_{i, j-1}^{t}}{(\Delta y)^{2}} \\
& +C \frac{n_{i+1, j+1}^{t}-n_{i+1, j-1}^{t}-n_{i-1, j+1}^{t}+n_{i-1, j-1}^{t}}{4 \Delta x \Delta y} \\
& +D \frac{n_{i+1, j}^{t}-n_{i-1, j}^{t}}{2 \Delta x} \\
& +E \frac{n_{i, j+1}^{t}-n_{i, j-1}^{t}}{2 \Delta y} \\
& +F n_{i, j}^{t}
\end{aligned}
$$

That we can also write:

$$
\begin{aligned}
n_{i, j}^{t+\Delta t} & =P_{0} n_{i, j}^{t}+P_{1} n_{i+1, j}^{t}+P_{2} n_{i-1, j}^{t}+P_{3} n_{i, j+1}^{t}+P_{4} n_{i, j-1}^{t} \\
& +P_{5} n_{i+1, j+1}^{t}+P_{6} n_{i-1, j-1}^{t}+P_{7} n_{i+1, j-1}^{t}+P_{8} n_{i-1, j+1}^{t}
\end{aligned}
$$

The displacement of the cell is determined by the following set of coefficients $P_{k}$ which relate to the probability for the cell to move in one of the 8 possible directions of the $2 \mathrm{D}$ grid (Fig. 2): 


$$
\begin{aligned}
& P_{0}=1-\frac{2 \Delta t}{(\Delta x)^{2}} A-\frac{2 \Delta t}{(\Delta y)^{2}} B+\Delta t F \\
& P_{1}=\frac{\Delta t}{(\Delta x)^{2}} A+\frac{\Delta t}{2 \Delta x} D \\
& P_{2}=\frac{\Delta t}{(\Delta x)^{2}} A-\frac{\Delta t}{2 \Delta x} D \\
& P_{3}=\frac{\Delta t}{(\Delta y)^{2}} B+\frac{\Delta t}{2 \Delta y} E \\
& P_{4}=\frac{\Delta t}{(\Delta y)^{2}} B-\frac{\Delta t}{2 \Delta y} E \\
& P_{5}=P_{6}=\frac{\Delta t}{4 \Delta x \Delta y} C \\
& P_{7}=P_{8}=-\frac{\Delta t}{4 \Delta x \Delta y} C
\end{aligned}
$$

The probability coefficients $P_{k}$ are evaluated from the chemotactic, haptotactic and strain-biased diffusion terms of equation $(2.2)$
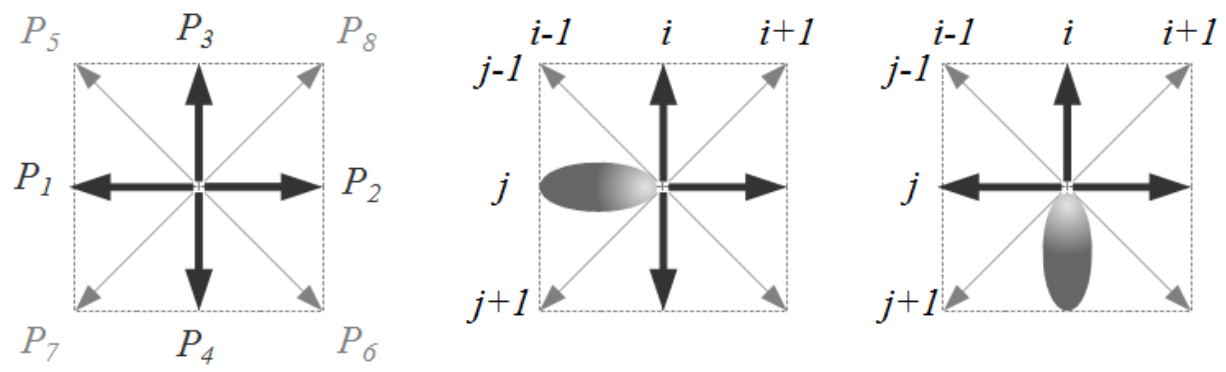

FiguRE 2. Representation of the 8 possible directions for cell displacement (left graph). The probability to move in one particular direction $k$ is given by the corresponding coefficient $P_{k} . \quad P_{0}$ which is the non-movement probability, is not indicated. The cell either occupies a horizontal segment (middle graph) or vertical segment (right graph) of the $2 \mathrm{D}$ simulation grid. The cell is also polarized and cannot move back.

\subsubsection{Derivation of each term}

Chemotactic term

$$
\begin{aligned}
\nabla \cdot(\chi(c) n \nabla c) & =\frac{\partial}{\partial x}\left[\chi(c) n \frac{\partial c}{\partial x}\right]+\frac{\partial}{\partial y}\left[\chi(c) n \frac{\partial c}{\partial y}\right] \\
& =\left[-\frac{\alpha \chi}{(1+\alpha c)^{2}}\left(\left(\frac{\partial c}{\partial x}\right)^{2}+\left(\frac{\partial c}{\partial y}\right)^{2}\right)+\frac{\chi}{1+\alpha c}\left(\frac{\partial^{2} c}{\partial x^{2}}+\frac{\partial^{2} c}{\partial y^{2}}\right)\right] n \\
& +\frac{\chi}{1+\alpha c} \frac{\partial c}{\partial x} \frac{\partial n}{\partial x}+\frac{\chi}{1+\alpha c} \frac{\partial c}{\partial y} \frac{\partial n}{\partial y}
\end{aligned}
$$


Haptotactic term

$$
\begin{aligned}
\rho \nabla \cdot(n \nabla f) & =\rho \frac{\partial}{\partial x}\left(n \frac{\partial f}{\partial x}\right)+\rho \frac{\partial}{\partial y}\left(n \frac{\partial f}{\partial y}\right) \\
& =\rho\left[\frac{\partial n}{\partial x} \frac{\partial f}{\partial x}+\frac{\partial n}{\partial y} \frac{\partial f}{\partial y}+n\left(\frac{\partial^{2} f}{\partial x^{2}}+\frac{\partial^{2} f}{\partial y^{2}}\right)\right]
\end{aligned}
$$

Strain-biased diffusion term

$$
\begin{aligned}
\nabla \cdot(\boldsymbol{D}(\varepsilon) n) & =\boldsymbol{D}(\varepsilon) \nabla n+n \nabla \cdot[\boldsymbol{D}(\varepsilon)] \\
\nabla \cdot[\nabla \cdot(\boldsymbol{D}(\varepsilon) n)] & =D_{0}\left(2+\varepsilon_{d}\right) \frac{\partial^{2} n}{\partial x^{2}}+D_{0}\left(2-\varepsilon_{d}\right) \frac{\partial^{2} n}{\partial y^{2}}+2 D_{0} \varepsilon_{s} \frac{\partial^{2} n}{\partial x \partial y} \\
& +2 D_{0}\left(\frac{\partial \varepsilon_{d}}{\partial x}+\frac{\partial \varepsilon_{s}}{\partial y}\right) \frac{\partial n}{\partial x}-2 D_{0}\left(\frac{\partial \varepsilon_{d}}{\partial y}-\frac{\partial \varepsilon_{s}}{\partial x}\right) \frac{\partial n}{\partial y} \\
& +D_{0}\left(\frac{\partial^{2} \varepsilon_{d}}{\partial x^{2}}-\frac{\partial^{2} \varepsilon_{d}}{\partial y^{2}}+2 \frac{\partial^{2} \varepsilon_{s}}{\partial x \partial y}\right) n
\end{aligned}
$$

with $\quad \varepsilon_{d}=\varepsilon_{x x}-\varepsilon_{y y} \quad$ and $\quad \varepsilon_{s}=2 \varepsilon_{x y}=2 \varepsilon_{y x}$

\subsubsection{Identification of the probability coefficients $\boldsymbol{P}_{\boldsymbol{k}}$}

The development of the continuous equation (2.2) on a $2 \mathrm{D}$ domain gives:

$$
\begin{aligned}
\frac{\partial n}{\partial t} & =D_{0}\left(2+\varepsilon_{d}\right) \frac{\partial^{2} n}{\partial x^{2}}+D_{0}\left(2-\varepsilon_{d}\right) \frac{\partial^{2} n}{\partial y^{2}}+2 D_{0} \varepsilon_{s} \frac{\partial^{2} n}{\partial x \partial y} \\
& +\left[2 D_{0}\left(\frac{\partial \varepsilon_{d}}{\partial x}+\frac{\partial \varepsilon_{s}}{\partial y}\right)-\frac{\chi}{1+\alpha c} \frac{\partial c}{\partial x}-\rho \frac{\partial f}{\partial x}\right] \frac{\partial n}{\partial x} \\
& -\left[2 D_{0}\left(\frac{\partial \varepsilon_{d}}{\partial y}-\frac{\partial \varepsilon_{s}}{\partial x}\right)+\frac{\chi}{1+\alpha c} \frac{\partial c}{\partial y}+\rho \frac{\partial f}{\partial y}\right] \frac{\partial n}{\partial y} \\
& +D_{0}\left(\frac{\partial^{2} \varepsilon_{d}}{\partial x^{2}}-\frac{\partial^{2} \varepsilon_{d}}{\partial y^{2}}+2 \frac{\partial^{2} \varepsilon_{s}}{\partial x \partial y}\right) n \\
& +\frac{\alpha \chi}{(1+\alpha c)^{2}}\left[\left(\frac{\partial c}{\partial x}\right)^{2}+\left(\frac{\partial c}{\partial y}\right)^{2}\right] n-\frac{\chi}{1+\alpha c}\left(\frac{\partial^{2} c}{\partial x^{2}}+\frac{\partial^{2} c}{\partial y^{2}}\right) n \\
& -\rho\left(\frac{\partial^{2} f}{\partial x^{2}}+\frac{\partial^{2} f}{\partial y^{2}}\right) n
\end{aligned}
$$

We now identify the coefficients of equation (2.17) with this resulting equation (2.30) to evaluate the migration probabilities $P_{k}$ :

$$
\begin{aligned}
P_{0} & =1-2 D_{0} \Delta t\left[\frac{2+\varepsilon_{d}}{(\Delta x)^{2}}+\frac{2-\varepsilon_{d}}{(\Delta y)^{2}}-\frac{1}{2} \frac{\partial^{2} \varepsilon_{d}}{\partial x^{2}}+\frac{1}{2} \frac{\partial^{2} \varepsilon_{d}}{\partial y^{2}}-\frac{\partial^{2} \varepsilon_{s}}{\partial x \partial y}\right] \\
& +\frac{\alpha \chi \Delta t}{(1+\alpha c)^{2}}\left[\left(\frac{\partial c}{\partial x}\right)^{2}+\left(\frac{\partial c}{\partial y}\right)^{2}\right]-\frac{\chi \Delta t}{1+\alpha c}\left(\frac{\partial^{2} c}{\partial x^{2}}+\frac{\partial^{2} c}{\partial y^{2}}\right) \\
& -\rho \Delta t\left(\frac{\partial^{2} f}{\partial x^{2}}+\frac{\partial^{2} f}{\partial y^{2}}\right)
\end{aligned}
$$




$$
\begin{aligned}
P_{1} & =\frac{D_{0} \Delta t}{(\Delta x)^{2}}\left(2+\varepsilon_{d}\right)+\frac{D_{0} \Delta t}{\Delta x}\left(\frac{\partial \varepsilon_{d}}{\partial x}+\frac{\partial \varepsilon_{s}}{\partial y}\right) \\
- & \frac{\chi}{(1+\alpha c)} \frac{\Delta t}{2 \Delta x} \frac{\partial c}{\partial x}-\rho \frac{\Delta t}{2 \Delta x} \frac{\partial f}{\partial x} \\
P_{2} & =\frac{D_{0} \Delta t}{(\Delta x)^{2}}\left(2+\varepsilon_{d}\right)-\frac{D_{0} \Delta t}{\Delta x}\left(\frac{\partial \varepsilon_{d}}{\partial x}+\frac{\partial \varepsilon_{s}}{\partial y}\right) \\
& +\frac{\chi}{(1+\alpha c)} \frac{\Delta t}{2 \Delta x} \frac{\partial c}{\partial x}+\rho \frac{\Delta t}{2 \Delta x} \frac{\partial f}{\partial x} \\
P_{3} & =\frac{D_{0} \Delta t}{(\Delta y)^{2}}\left(2-\varepsilon_{d}\right)-\frac{D_{0} \Delta t}{\Delta y}\left(\frac{\partial \varepsilon_{d}}{\partial y}-\frac{\partial \varepsilon_{s}}{\partial x}\right) \\
& -\frac{\chi}{(1+\alpha c)} \frac{\Delta t}{2 \Delta y} \frac{\partial c}{\partial y}-\rho \frac{\Delta t}{2 \Delta y} \frac{\partial f}{\partial y} \\
P_{4} & =\frac{D_{0} \Delta t}{(\Delta y)^{2}}\left(2-\varepsilon_{d}\right)+\frac{D_{0} \Delta t}{\Delta y}\left(\frac{\partial \varepsilon_{d}}{\partial y}-\frac{\partial \varepsilon_{s}}{\partial x}\right) \\
& +\frac{\chi}{(1+\alpha c)} \frac{\Delta t}{2 \Delta y} \frac{\partial c}{\partial y}+\rho \frac{\Delta t}{2 \Delta y} \frac{\partial f}{\partial y} \\
P_{5} & =P_{6}=\frac{D_{0} \Delta t}{2 \Delta x \Delta y} \varepsilon_{s} \\
P_{7} & =P_{8}=-\frac{D_{0} \Delta t}{2 \Delta x \Delta y} \varepsilon_{s}
\end{aligned}
$$

We note that the probabilities along the diagonal directions are all equivalent and are moreover very small compared to the other orthogonal directions. We will therefore only consider cell migration along the horizontal and vertical main directions by re-normalizing the probabilities of these four directions.

\subsection{Algorithm}

Figure 3 presents the model implementation and the inter-relations between the molecular and mechanical components.

1. a few cells (sprouts) are initially positioned at some locations $(i, j)$ onto the simulation domain for which $n_{i, j}=1$ and 0 everywhere else (no cells);

2. enzymes $(m)$, growth factors $(c)$, and matrix fibres $(f)$ concentrations are then updated (by solving eq.(2.3),(2.4),(2.5)) since their production / consumption / degradation depend on the presence of "active" cells (i.e. the sprouts);

3. the Young modulus $E(f)$ can be updated since it directly depends on the matrix fibres concentration $(f)$;

4. traction forces $\left(\tau n_{i, j} \boldsymbol{e}\right)$ are applied at specific locations on the extracellular matrix identified by the cells positions $n_{i, j}=1$ and cells orientations $\boldsymbol{e}$;

5. matrix displacements $u$ and $v$ can be calculated (from eq.(2.15) and (2.16)) based on the updated matrix rigidity and traction forces;

6. strains $(\varepsilon)$ in the matrix are directly derived from those displacements with eq.(2.14); 


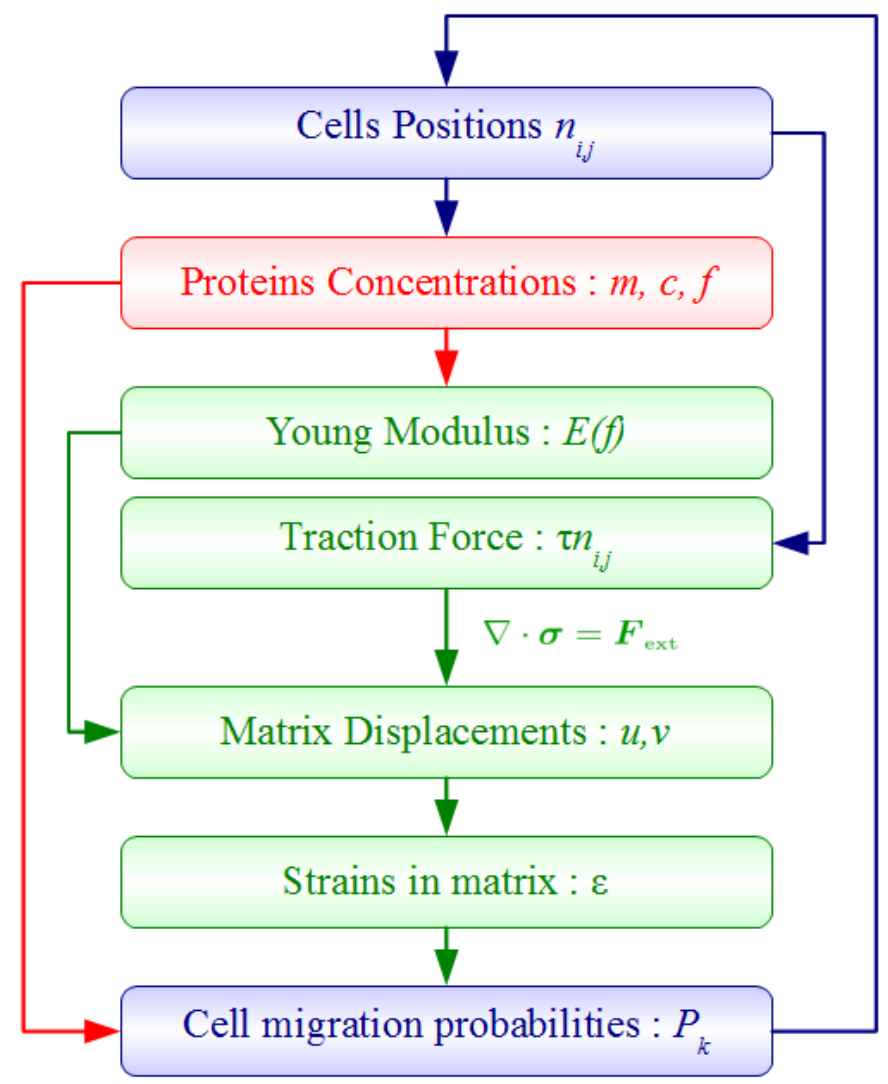

FIgURE 3. Graph showing the model implementation with its successive calculation steps. The red box refers to the molecular component and the green boxes to the mechanical components.

7. cell migration probabilities in eq.(2.31) to (2.37) are evaluated from the contributions of the molecular and mechanical components;

8. finally, the new cell positions are updated.

Important remark: In this paper, we use arbitrary units. We make no attempt to link our parameters (Young's modulus, cell traction force intensity, etc.) and simulated variables (fibre density, cell velocity, matrix deformations, etc.) to known values. Our aim, at this stage, is to qualitatively appreciate the contribution of the various mechanisms. This appreciation is therefore purely relative from one measurement to another. All parameters used for the simulations are given in appendix B.

\section{Simulation Results}

\subsection{Consequence of the mechanical input on angiogenesis}

In these simulations we want to test the contribution of mechanical cues, specifically the cell sensitivity to the strains in the matrix generated by the traction forces developed by the migrating cells themselves. Simulations are performed for different values of the traction force coefficient $\tau$. 
For each values of $\tau$ one hundred simulations are performed. As initial condition, we consider a homogeneous distribution of matrix fibres and a steady state gradient of growth factors corresponding to the solution of the diffusion equation with a virtual tumour source located on the right hand side of the simulation domain. Five sprouts are initiated at the opposite side of the domain. For the sake of simplicity, growth factors diffusion is then neglected in the simulations $\left(D_{c}=0\right)$, since we focus on its chemoattracting property rather than than on its full dynamics. Each simulation stops when there are no more sprouts (sprouts disappear when they hit a boundary or when they anastomose with another sprout or vessel). The one hundred simulated vascular networks are then superimposed using a colour code to highlight the density of the trajectories in the domain (Fig. 4A). Figure 4 presents the simulation results, where for each value of the parameter $\tau$, one example of a characteristic vascular network is shown (Fig. 4B) with its corresponding matrix degradation map (Fig. 4C).

We observe that, as the cell traction force increases, the vascular networks become more "organised", in the sense that the vessels are less tortuous and the density of vessels is lower. However this is true up to $\tau=20 e 4$ above which the vascular networks cannot form any more. The vascular network formation is accompanied by the degradation of the matrix due to the migrating endothelial cells. The size of the surface affected by proteases degradation is bigger when the cells moves more randomly since they explore a larger surface and this happens when cells are not directed by the mechanical cues (Fig. 4C, $\tau=0$ ). This effect has been quantified over 100 simulations for each value of the traction force intensity $\tau$ and the results are presented in Figure 5. We observe that when the cell traction force is not considered $(\tau=0)$, almost $20 \%$ of the matrix surface is degraded above $30 \%$ of its original density. Even when the traction force is weak $(\tau=10 e 4)$, differences in the degradation profile are already visible with less than $10 \%$ of the matrix surface degraded above $30 \%$. For a traction force intensity above $\tau=20 e 4$ the matrix degradation level does not exceed $20 \%$ any more and the formation of a functional vascular network is strongly impeded.

Figure 6 presents some characteristics of the vascular networks measured from 100 simulations for each case. Specifically the network length, the number of anastomoses and branches are represented. As the cell traction forces exerted on the extracellular matrix increases the total network length decreases with less branches formed and therefore less connections between branches which corresponds to the anastomosis process. Since the number of anastomoses is strongly related to the number of branches formed, the ratio of these two numbers is also plotted and highlight two phases: first, a decrease of this ratio indicates that more branches are formed than loops and from $\tau=20 e 4$ an increase of the ratio above 1, indicates that there are more loops than branches since the vessels fold on themselves and lose the ability to branch. Loops are required to allow the blood to flow through the vessels. However, two many loops impede the efficiency of the blood flow since they are the cause of aberrant and tortuous tumour vascular networks [36]. So the number of branches should ideally equilibrate with the number of loops with an optimum ratio to get a functional network. The network is functional as long as the number of branches is higher than the number of loops, since two branches should meet to make one loop.

In this first series of simulations, it is difficult to isolate the mechanical effects on the resulting vascular structure since the gradient of growth factors is also playing a part in stimulating the formation of branches in a spatially non-homogeneous way.

In order to avoid this constraint, we removed the spatial distribution of growth factor so that the formation of branches is spatially homogeneously stimulated. This implies that the network is not growing directionally any more toward a target such as a tumour. So this next series of simulations is a case study which is not directly related to a biological reality but that we use to highlight in a better way the effects of the integration of the mechanical aspects. 


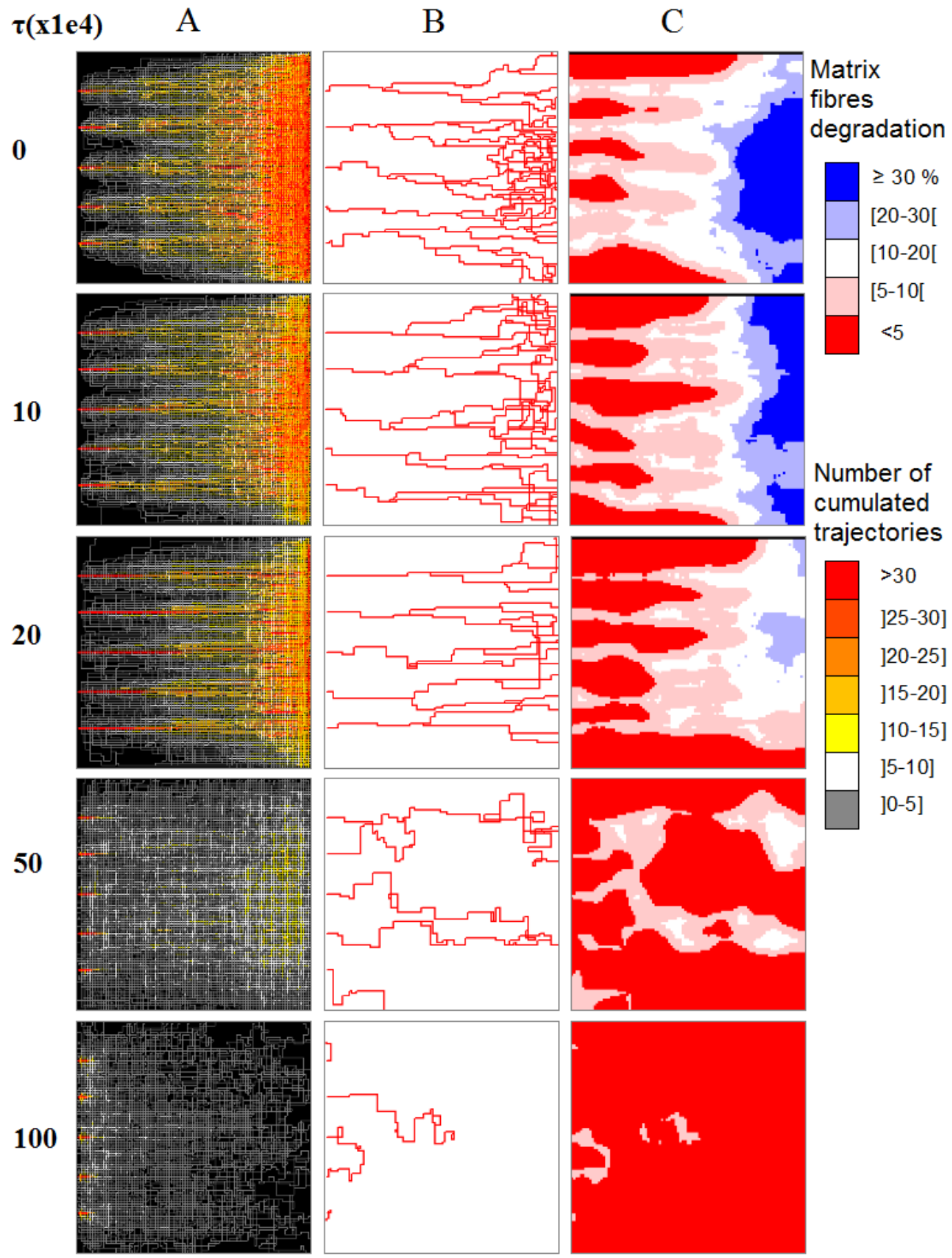

FiguRE 4. Simulations of network growth. (A) 100 superimposed networks, where the colour code indicates the number of trajectories that passes through each segment of the grid; (B) representative networks for each value of the parameter $\tau$ and (C) their corresponding matrix degradation map (\% degraded). 


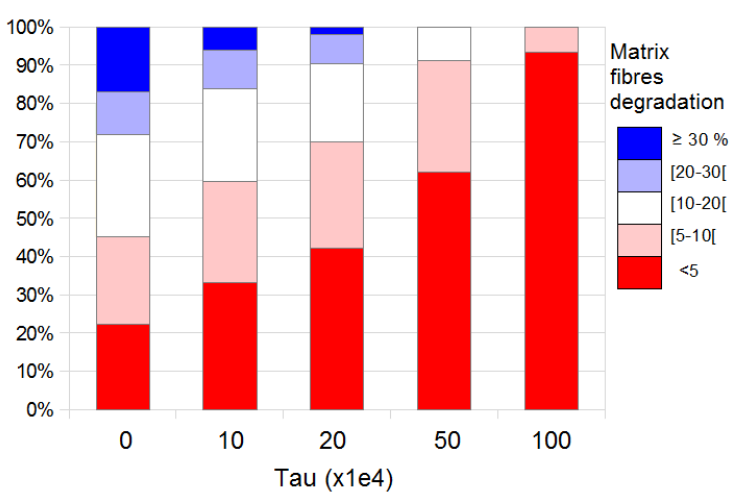

FiguRE 5. Mean ratio of the surface degraded at the different levels of degradation from low (red) to high (blue) for different values of the cell traction force $\tau$. Mean values are evaluated from 100 simulations.
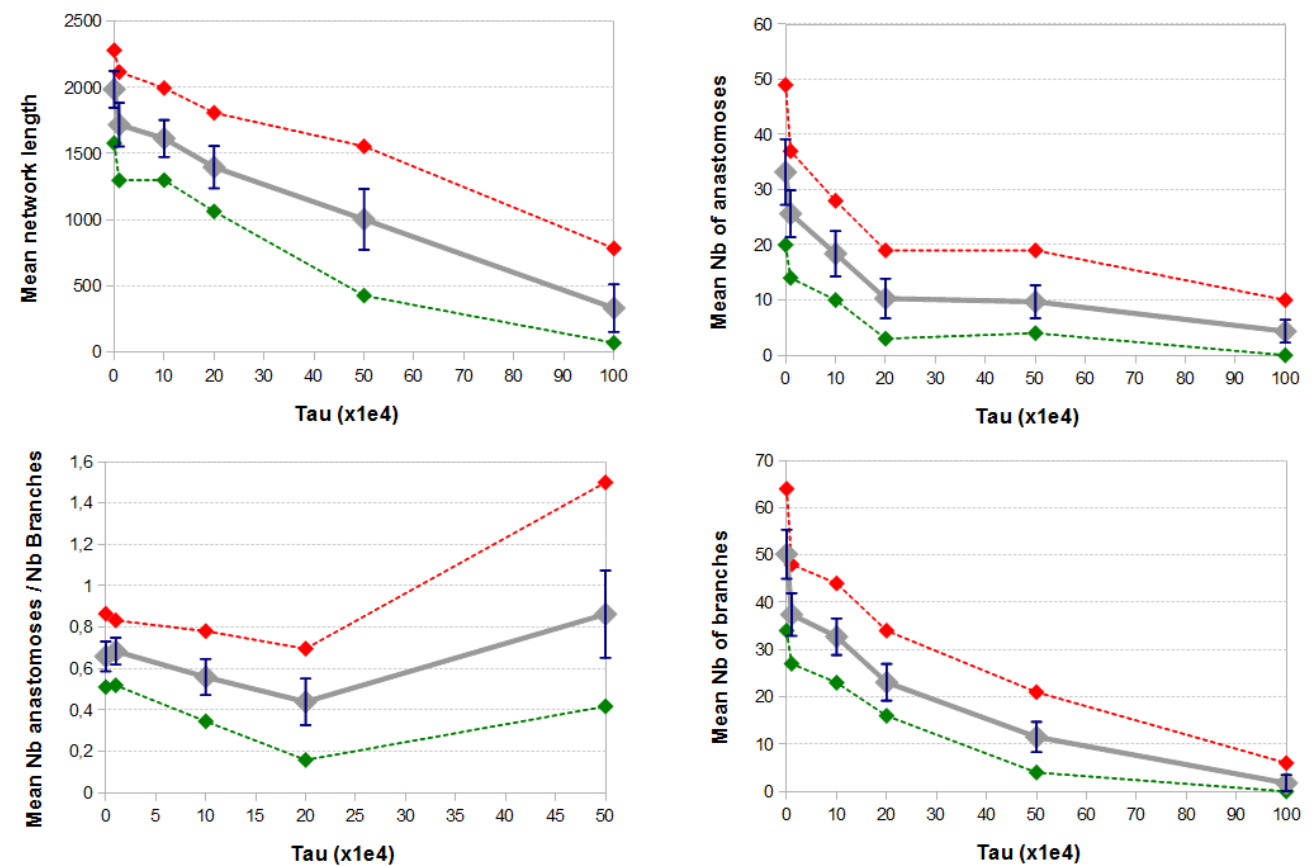

FiguRE 6. Evolutions of the mean network length, mean number of anastomoses and mean number of branches as a function of the traction force intensity (grey curves). The dotted red and green curves represent the maximum and minimum values respectively obtained over 100 simulations for each point.

We reproduce the simulations realised in the first series by removing the gradient of growth factor which means that the chemotactic cue is removed (which is also equivalent to $\chi=0$ ). However we keep a homogeneous distribution of this growth factor so as to stimulate the formation of branches in a spatially homogeneous way: the probability for a vessel to make a branch is the same everywhere in the simulation domain. 
Since there is no gradient to lead the vessels growth direction, the initial sprouts are placed one in each quarter of the domain with alternate orientations so as to favour a homogeneous exploration of the environment (Fig. 7).
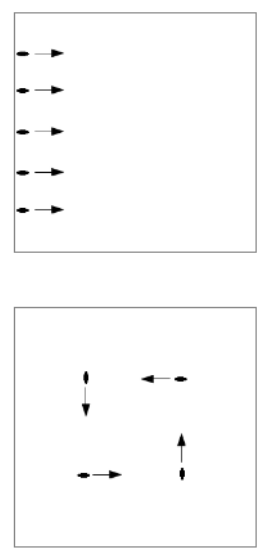

to

t1
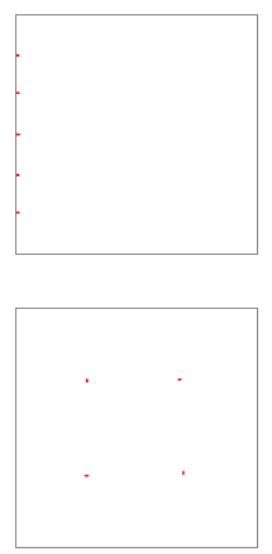

t2
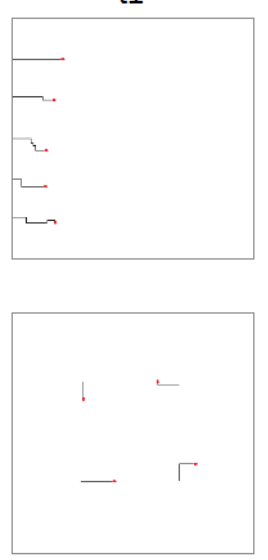
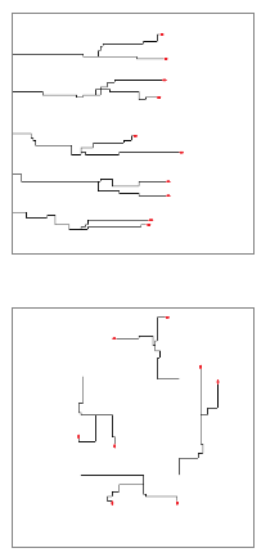

t3
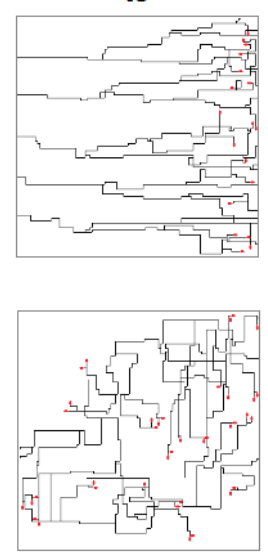

Figure 7. Positions and orientations of the initial sprouts for the two series of simulations corresponding to figure 4 (upper panel) and to figure 8 (lower panel). Three consecutive times of the simulations for $\tau=10 e 4$ are shown. The red spots correspond to the sprouts.

As in the first series, 100 simulations are performed for each values of the traction force coefficient $\tau$. The structures generated are superimposed to visualize the spatial density of the cell trajectories in the simulation domain (Fig. 8A). As previously, an example of the structure generated for each case is presented (Fig. 8B) with its corresponding degradation map (Fig. 8C).

The simulation results presented in Figure 8 show that as the cell traction force increases from $\tau=0$ to $20 \mathrm{e}$, the vessels become more rectilinear and the density of the generated structure decreases as attested by the degradation profile (Fig. 9) and the decrease of the total network length (Fig. 10, top graph). As in the first series, above the same value of the traction force $(>\tau=20 e 4)$ no connected structures can be generated any more. The cell migration is tortuous again and loops are formed before the vessels had time to branch (Fig. 10).

This second series of simulations confirms that the cell traction force can be both an organizing force to generate well structured networks or a disruptive force when its intensity is too strong. We note that the intensity of $\tau$ is translated into a strain on the matrix. This strain is contributing in the probability coefficients for migration. If $\tau$ is too high, the strain is also high and the cell is "trapped" in the strain field in a small perimeter around the tip cell as if the cell was self-attracted, hence the loop kills the sprout before it had time to branch and this prevents the formation of a network.

\subsection{Cell sensitivity to the mechanical environment}

In the following simulations we aim to decipher the contributions of the two main mechanical parameters in this model which are the traction force intensity $(\tau)$ of the cell as it migrates and the rigidity (Young's modulus $E$ ) of the fibre matrix. 


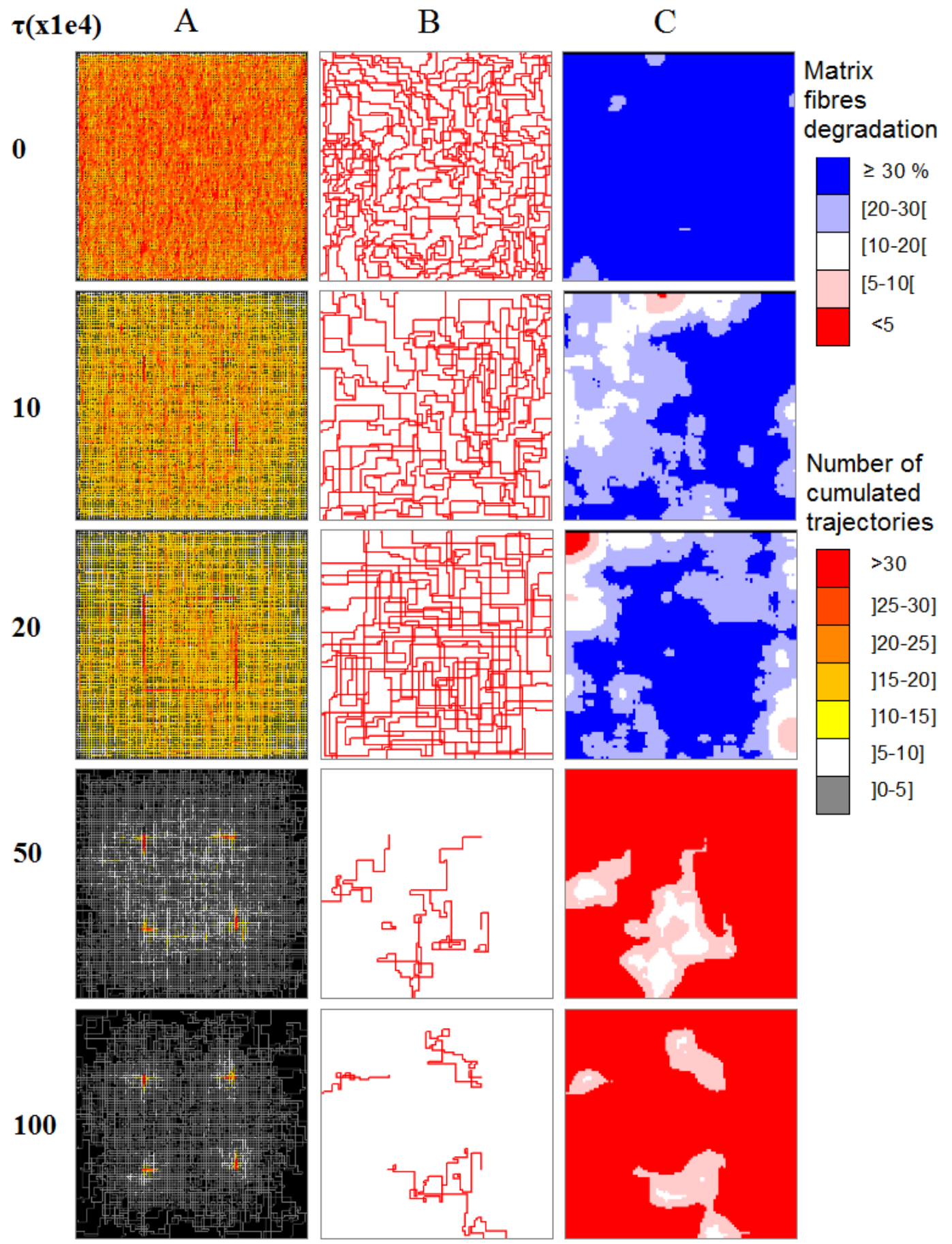

FiguRE 8. Simulations of network growth. (A) 100 superimposed networks, where the colour code indicates the number of trajectories that passes through each pixel; (B) representative networks for each value of the parameter $\tau$ and $(\mathrm{C})$ their corresponding matrix degradation map (\% degraded). 


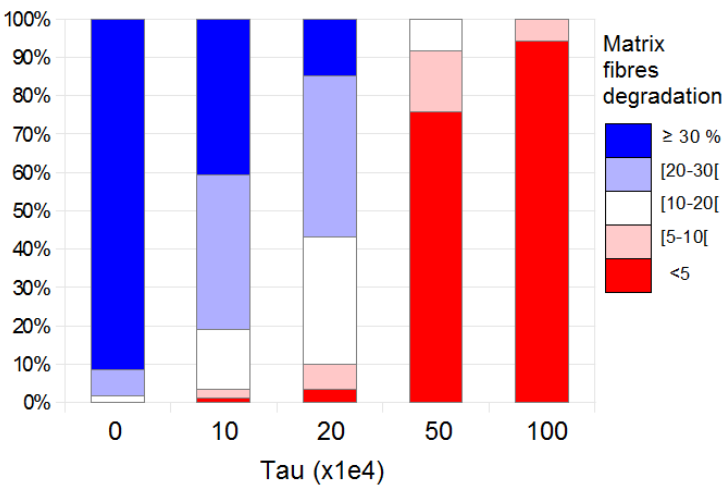

FiguRE 9. Mean ratio of the surface degraded at the different levels of degradation from low (red) to high (blue) for different values of the cell traction force $\tau$. Mean values are evaluated from 100 simulations.
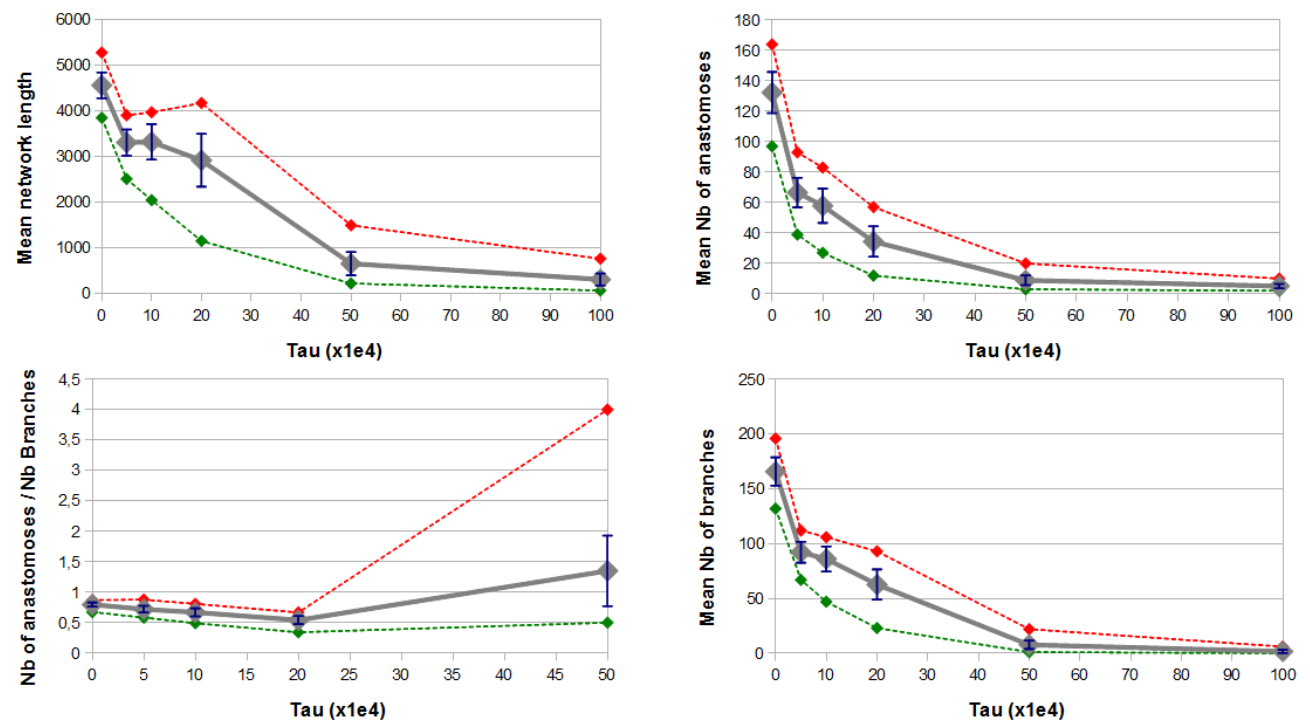

FigURE 10. Evolutions of the mean network length, mean number of anastomoses and mean number of branches as a function of the traction force intensity (grey curves). The dotted red and green curves represent the maximum and minimum values respectively obtained over 100 simulations for each point.

\subsubsection{Influence of the matrix rigidity}

In the simulations previously made, the migrating cells were allowed to degrade the fibre matrix. The degraded surface could be vast and profound, above $30 \%$ of the initial fibre matrix density. Although it is clear from those results that the traction force intensity influences the cell migration and the resulting structures (vascular network), it is not yet clear if changes in the matrix rigidity are also playing a role.

We made a series of new simulations where a single cell is placed at the centre of the domain. The matrix fibre density is homogeneous and there are no sources of growth factors, which means there is no chemoattraction. The cell is, this time, not allowed to degrade the matrix $(\gamma=0)$, so there is no haptotaxis either. The cell migrates randomly and it cannot divide to form branches. We record the 
cell trajectory and measure the tortuosity and velocity of this trajectory for different matrix rigidities (Young's modulus $E$ ) and different cell traction force intensities $(\tau)$. One hundreds trajectories are simulated for each $(E, \tau)$ couple.

The tortuosity index is calculated as the ratio between the length of the straight line from the starting to arrival points (when the cell hits one boundary of the domain) and the total length of the trajectory. This means that an index equals to one corresponds to a perfectly straight trajectory. The smaller the index, the more tortuous the trajectory. The mean cell velocity therefore corresponds to the total length of the trajectory divided by the time it takes to go all over it.

Figure 11 confirms that the traction force intensity has an impact on the characteristics of the trajectories. Specifically, the upper graph shows that, as the traction force intensity increases, the velocity of the trajectories increases significantly. The tortuosity of the trajectories is also impacted (lower graph) with a higher tortuosity index, indicating more directed trajectories for a smaller traction force intensity. However the decrease in the tortuosity index is not significantly different for $\tau=5 e 5$ and $\tau=10 e 5$ where it saturates around the value of 0.10 (this saturation value is related to the size of the domain).

Interestingly, small velocities are associated with more rectilinear trajectories, which is somehow counter-intuitive. We would expect that changing trajectories would cost more in time than keeping the same direction. In this case, it appears that the environmental constraints (strains) prevent the cell from changing direction (i.e. the probability for no movement $P_{0}$ is selected more often), so the resulting directed trajectory is not the "easiest" path but the most constrained. In other words it can be seen as an impeded trajectory, whereas the most random trajectories are constraint-free and more rapid.

We then evaluate the influence of changes in the matrix rigidity (Young's modulus, $E$ ) by selecting a range of rigidity values compatible with a degradation depth of about $30 \%$. (since $E$ linearly depends on the density of matrix fibres, it is altered in the same proportion. A fibres degradation of $30 \%$ approximatively corresponds to a change in $E$ from 15 to 10). The influence of changes in $E$ is quite small especially on the velocity. Only a slight decrease of the velocity is observed as $E$ increases in the range of considered values. The effect of the changes in $E$ are the most important for the tortuosity index corresponding to a traction force intensity $\tau=2 e 5$. In this case, the trajectories are even more directed with increasing rigidity, although the standard deviation from the mean value is high. The most directed trajectories are observed for the couple of parameters $(E, \tau)=(15,2 e 5)$.

Since the effect the matrix rigidity is most prominent for $\tau=2 e 5$, the spectre of rigidity values has been extended for this specific traction force intensity. Figure 12 shows that the velocity remains high and almost constant from $E=0.001$ to 1 and then decreases sharply from $E=1$ to 100 . Effects on the tortuosity are located in the same range for $E$, but with a biphasic evolution. The tortuosity first increases for $E=1$ to 10 and then decreases for $E=10$ to 100 . However from figure 11, we know that the tortuosity index is even higher for $E=15$. This means that the maximum is somewhere between $E=15$ and $E=100$.

\subsubsection{Consequence on the matrix deformations}

For each couple of parameters $(E, \tau)$ we simulated the matrix deformations (strains) for a single non-migrating cell placed horizontally (i.e. oriented along $x$ ) in the centre of the simulation domain. The graphs presented in Figure 13, exhibit the maximum strain measured $\left(\varepsilon_{x x}\right)$ as a function of $E$ (or $\log E)$. First, the maximum strain decreases as the matrix rigidity $E$ increases. Second, the maximum strain increases as the cell traction force intensity $\tau$ increases. These two facts are coherent with known experimental data [22]. On the wider range of rigidities, we observe that the matrix deformation 

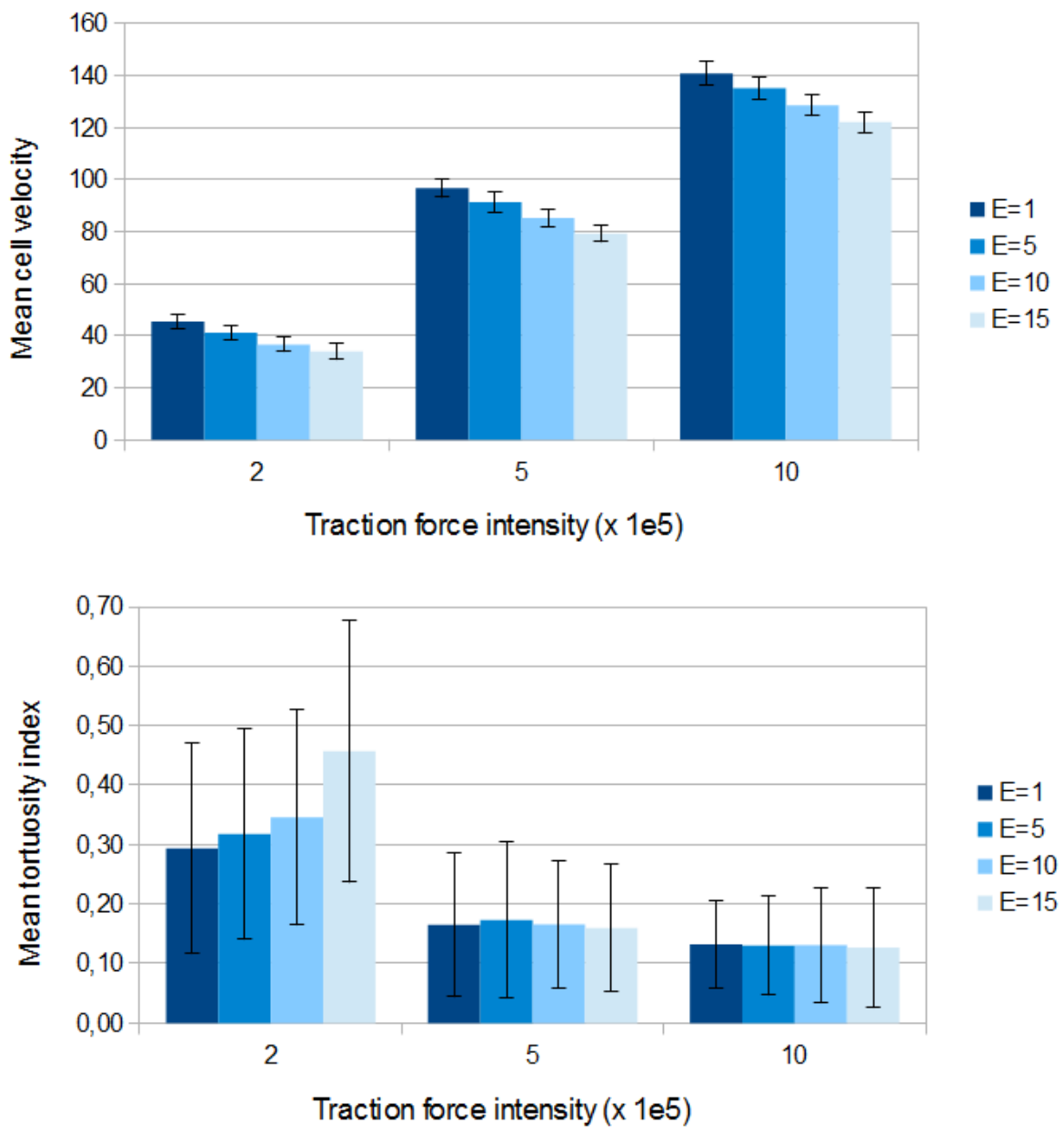

FIgURE 11. Mean cell velocity (arbitrary unit) and mean tortuosity index for different matrix rigidities and traction force intensities.

saturates to their highest value for very compliant matrices (no resistance to the deformation). Then the deformation starts to decrease with increasing rigidity (increased resistance to deformation).

We note that the highest velocity for cell migration correspond to the highest strain. But this high deformation does not enhance directionality in cell migration (low tortuosity index).

\subsection{Conclusion}

Our results show that acting on the mechanics can significantly alter cell migration and angiogenesis. First, there is a limited range of traction force intensities for which a vascular network can be obtained. Second, the matrix rigidity plays a role, but only in a very specific range, compatible with the underlying biological process. Moreover changes of about $30 \%$ in the rigidity, which correspond to the alteration due to cell degradation, are too small to induce significant changes in cell migration trajectories (in terms of persistence of the migrating direction and velocity). Which means that in its actual form our model probably underestimates the mechanical importance of matrix degradation. 

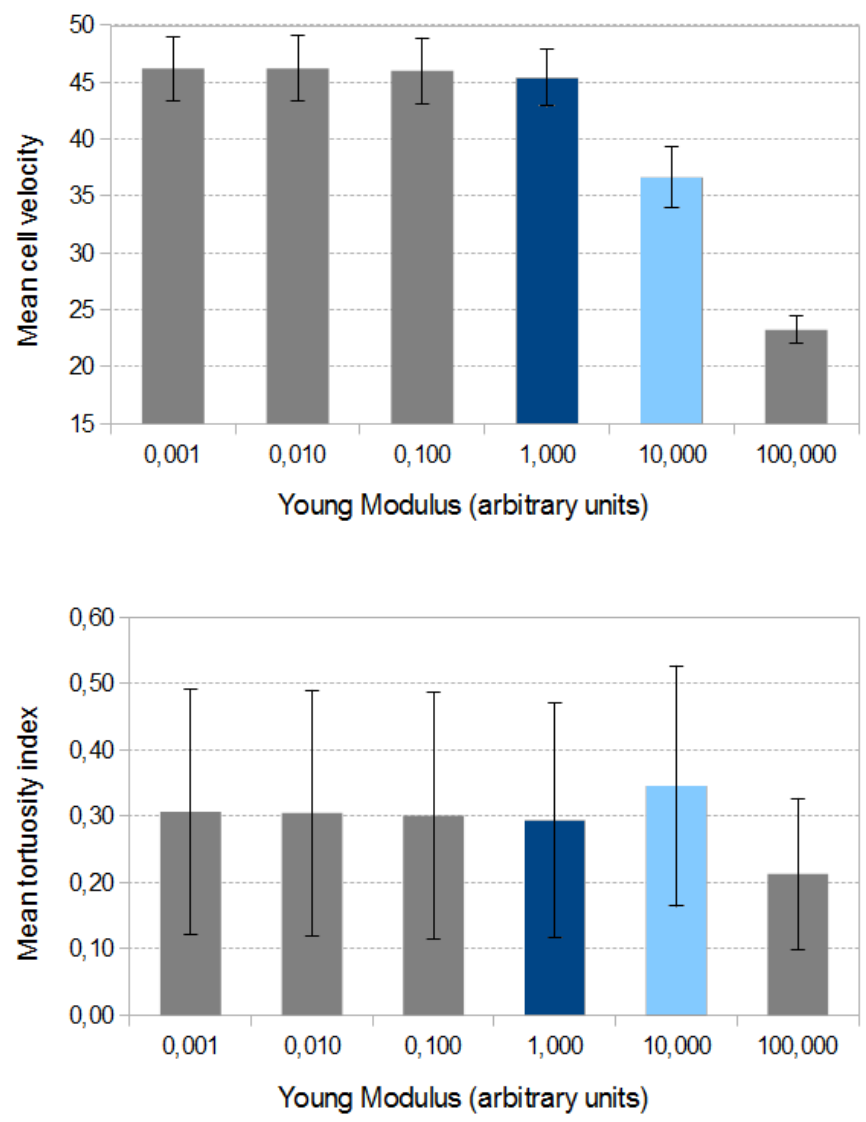

Figure 12. Mean cell velocity (arbitrary unit) and mean tortuosity index for an extended rang of matrix rigidities for the traction force intensity $\tau=2 e 5$. The blue boxes correspond to the values in figure 11.

\section{Discussion}

In this paper, we proposed a new hybrid model of angiogenesis which integrates mechanical aspects, including the cell traction force, the changing (time-dependent) mechanical properties of the matrix considering a non constant Young's modulus and the matrix deformations. At this stage, our aim was to determine if mechanics had an influence on angiogenesis with regards to the other molecular chemotactic and haptotactic processes and, if so, in what way.

For that, we first simulated angiogenesis, for different values of the cell traction force and showed that mechanics affects the structure of the resulting vascular network. For an increasing traction force, we found that the density of the vascular network decreases as observed by [4] and the vessels are also more rectilinear. The resulting vasculature appears more structured, but if the traction force is too high, no networks are able to form. We then looked more closely at the influence of the matrix rigidity (Young's modulus) in a range of values that allows to assess the differences induced by the degradation with changes in rigidity of about $30 \%$. This influence is evaluated on the tortuosity index (or persistence) and velocity of the trajectories. The role of the rigidity appears limited for small changes in $E$, but still compatible with known experimental data [22], since the increased matrix rigidity indeed corresponds to a slower cell velocity. We had to subsequently change the range of values of $E$ to observe clear differences 

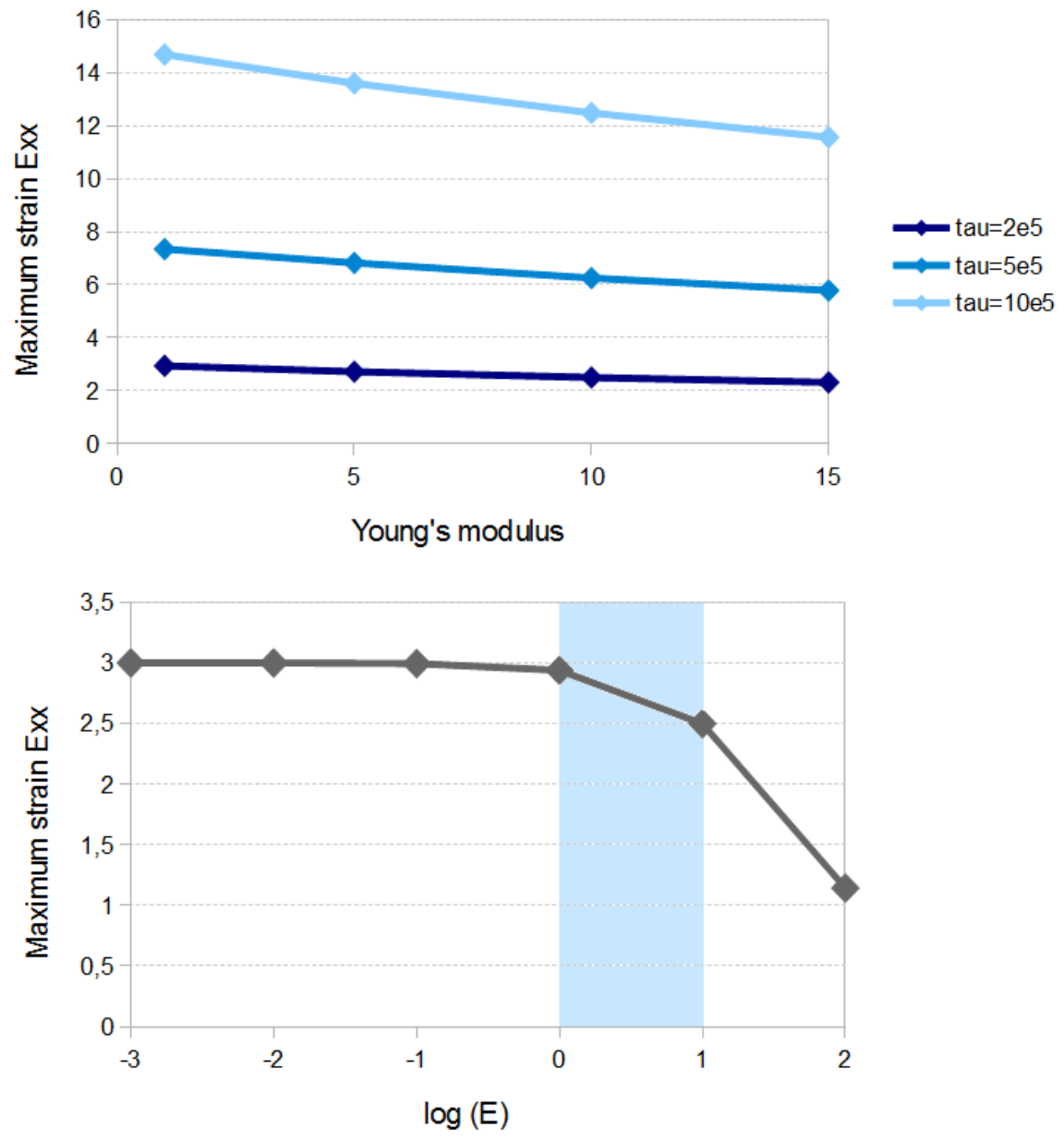

FiguRE 13. Maximum strain (\%) depending on the Young's modulus simulated for a single cell for the three traction forces intensities $\tau=2 e 5,5 e 5,10 e 5$ (upper graph). Maximum strain (\%) for a traction force intensity $\tau=2 e 5$ for a larger range of rigidity. The blue strip highlights the zone of values corresponding to the upper graph for $\tau=2 e 5$.

on the characteristics of the cell trajectories.

In this study, we considered arbitrary units, for all the parameters and variables, making no attempts to correlate those with real quantitative data. However, if we did (and we intend to do it in a very near future), it would be possible to identify optimum couples of parameters $(E, \tau)$ required to obtain given angiogenic patterns. But before that, many of our working assumptions will need to be revised and improved at the light of the most recent knowledge. The model was built from other existing models and the many terms were integrated as such. Other terms were also very crudely incorporated.

- In particular, the relationship between the Young's modulus and the fibre density which is purely linear needs to be better adjusted: $E$ does not exclusively depend on the fibres and it is required to account for limiting effects (for very high and very low fibre densities).

- To simplify the model equations, we considered an elastic matrix instead of a viscoelastic one which is more realistic to describe tissue behaviours. 
- The traction force intensity is considered constant but this is clearly not the case. It is well known that the cell adjusts its pulling force on the matrix to the rigidity [22]. For example, [41] considered a mechanosensing variable to adjust the traction stress to the ECM density.

- We could also test another diffusion tensor to better represent cell diffusive migration through the matrix.

- The body force, in the mechanical equilibrium, is not really necessary. It comes from previous models describing in vitro angiogenesis experiments to account for the attachment of the matrix to the Petri dish. But in our case, although we are considering a 2D tissue, this term is not reflecting any real contributing effect and could be removed.

- Last important remark, in this model cells are considered as individual entities, however we have not treated the extracellular matrix as a collection of individual fibres but rather as a continuum of varying fibre density. In this respect, the strain generated by individual cell and calculated "underneath" the cell is inaccurate since the matrix cannot be considered as a continuum at such a small scale (i.e. at the cell scale). However the strain evaluated in the small perimeter around the cell becomes acceptable. We intend to be more careful on the way the different scales are treated to describe properly the cell-matrix interaction in the sequel of this model, as this was done in models of cell migration $[6,7]$.

"Cell traction is the most important parameter controlling the deformation of the matrix and therefore angiogenic growth and remodeling" as pointed out by [11] and confirmed by our model simulations. Thus, taking into account mechanics is crucial to better understand cell migration in tissues and specifically angiogenesis, in order to control it and to derive some new therapies. This is especially important in the current context of cancer where more and more studies contribute to show that a tight regulation of the environment could be one key to help solve the cancer issue $[18,19,42]$. The actual trend is to define new therapies that act on the tumour environment $[17,28]$. One aim is to restore homeostasis so as to renormalize cancer cells and prevent or reverse cancer spreading [5].

Appendix

\section{A. Point Successive over-relaxation (SOR) method}

The point SOR method is an iterative method used to solve elliptic equations. These equations can be discretized on the grid $(i, j)$, for the general variable $W(x, y, t)$, under the form:

$$
A_{i} W_{i-1, j}+B_{i} W_{i, j}+C_{i} W_{i+1, j}+E_{i} W_{i, j-1}+F_{i} W_{i, j+1}+G_{i, j}=0
$$

\section{Algorithm}

The algorithm consists in choosing an arbitrary initial solution and to repeat the iterations until the convergence criteria is achieved.

while

$$
\begin{aligned}
\epsilon & <\sqrt{\left|W_{T}\right|^{2}(\Delta r)^{2}} \quad \text { (convergence criteria) } \\
W_{T} & =\frac{1}{B_{i}}\left[A_{i} W_{i-1, j}+C_{i} W_{i+1, j}^{k-1}+E_{i} W_{i, j-1}+F_{i} W_{i, j+1}^{k-1}+G_{i, j}\right]-W_{i, j}^{k-1} \\
W_{i, j} & =W_{i, j}^{k-1}+\omega W_{T}
\end{aligned}
$$

$\omega$ is the (over)relaxation coefficient taken between 1 and 2 (condition for convergence of the method). We take $\omega=1.25$. $W_{i, j}^{k-1}$ designates the $k-1$ (previous) virtual timestep. 


\section{B. Simulation parameters}

Parameters are in arbitrary units.

\begin{tabular}{cccc}
\hline Parameter & Significance & Value & Equation \\
\hline$D_{0}$ & Endothelial cell diffusion & 0.00035 & 2.2 \\
$\chi$ & Chemotactic coefficient & 0.38 & 2.2 \\
$\rho$ & Haptotactic coefficient & 0.34 & 2.2 \\
$\alpha$ & Cell sensitivity to growth factors & 0.6 & 2.2 \\
$D_{c}$ & Growth factor diffusion & 0.1 & 2.3 \\
$\eta$ & Growth factors consumption rate by cells & 0.1 & 2.3 \\
$\gamma$ & Matrix degradation rate by enzymes & 0.5 & 2.4 \\
$D_{m}$ & Enzymes diffusion & 0.1 & 2.5 \\
$\beta$ & Production rate of enzymes & $5 e 3$ & 2.5 \\
$\delta$ & Decay rate of enzymes & 50 & 2.5 \\
$s$ & Binding parameter of cell to the matrix & $1 e 6$ & 2.6 \\
$\tau$ & Cell traction force intensity & $10-100 e 4$ & 2.6 \\
$\nu$ & Poisson's ratio & 0.4 & 2.7 \\
$\kappa$ & Rigidity coefficient of the matrix fibres & $3.333-50$ & 2.7 \\
$f_{0}$ & Initial concentration of matrix fibres & 0.3 & \\
\hline
\end{tabular}

Acknowledgements. AC's research project on cell-matrix interactions is partially supported by the Rhône Alpes Complex Systems Institute (IXXI grant) and the University Joseph Fourier (AGIR grant).

\section{References}

[1] F. Amyot, A. Small, H. Boukari, K. Camphausen, A. Gandjbakhche. Topology of the heterogenous nature of the extracellular matrix on stochastic modeling of tumor-induced angiogenesis. Microvasc. Res., 77 (2009), 87-95.

[2] A.R.A. Anderson, M.A.J. Chaplain. Continuous and discrete mathematical models of tumor-induced angiogenesis. Bull. Math. Biol., 60 (1998), 857-900.

[3] A.L. Bauer, T.L. Jackson, Y. Jiang. A cell-based model exhibiting branching and anastomosis during tumor-induced angiogenesis. Biophys. J., 92 (2007), 3105-3121.

[4] Y. Cai, K. Gulnar, H. Zhang, J. Cao, S. Xu, Q. Long. Numerical simulation of tumor-induced angiogenesis influenced by the extra-cellular matrix mechanical environment. Acta Mech. Sin., 25 (2009), 889-895.

[5] J.P. Capp. Nouveau regard sur le cancer, pour une révolution des traitements. Ed. Belin (2012), ISBN 978-2-70115614-9.

[6] A. Chauvière, T. Hillen, L. Preziosi. Modeling cell movement in anisotropic and heterogeneous network tissues. Net. Het. Med., 2 (2007), 333-357.

[7] A. Chauvière, L. Preziosi. Mathematical framework to model migration of cell population in extracellular matrix. (2010) Cell Mechanics: From single-scale based models to multiscale modeling Taylor \& Francis Group, Chapman \& Hall/CRC, ISBN 978142009454.

[8] A. Das, D. Lauffenburger, H. Asada, R.D. Kamm. A hybrid continuum-discrete modelling approach to predict and control angiogenesis: analysis of combinatorial growth factor and matrix effects on vessel-sprouting morphology. Phil. Trans. R. Soc. A, 368 (2010), 2937-2960.

[9] G.E. Davis, D.R. Senger. Endothelial extracellular matrix. Biosynthesis, remodeling, and functions during vascular morphogenesis and neovessel stabilization. Circ. Res., 97 (2005), 1093-1107.

[10] J.T. Daub, R.M.H. Merks. A cell-based model of extracellular-matrix-guided endothelial cell migration during angiogenesis. Bull. Math. Biol., 75 (2013), 1377-1399.

[11] L.T. Edgar, J.B. Hoying, U Utzinger, C.J. Underwood, L. Krishnan, B.K. Baggett, S.A. Maas, J.E. Guilkey, J.A. Weiss. Mechanical interaction of angiogenic microvessels with the extracellular matrix. J. Biomech. Eng., 136 (2014), 021001.

[12] L.T. Edgar, S.C. Sibole, C.J. Underwood, J.E. Guilkey, J.A. Weiss. A computational model of in vitro angiogenesis based on extracellular matrix fibre orientation. Comp. Meth. Biomech. Biomed. Eng., 16 (2013), 790-801.

[13] L.T. Edgar, C.J. Underwood, J.E. Guilkey, J.B. Hoying, J.A. Weiss. Extracellular matrix density regulates the rate of neovessel growth and branching in sprouting angiogenesis. PLOS ONE, 9 (2014): e85178.

[14] J. Folkman, C. Haudenschild. Angiogenesis in vitro. Nature, 288 (1980), 551-556.

[15] H. Gerhardt. VEGF and endothelial guidance in angiogenic sprouting. Organogenesis, 4 (2008): 241-246.

[16] M.J. Holmes, B.D. Sleeman. A mathematical model of tumour angiogenesis incorporating cellular traction and viscoelastic effects. J. Theor. Biol., 202 (2000), 95-112. 
[17] D.E. Ingber. Can cancer be reversed by engineering the tumor microenvironment? Sem. Canc. Biol. 18 (2008), $356-364$.

[18] P. Katira, R.T. Bonnecaze, M.H. Zaman. Modeling the mechanics of cancer: effect of changes in cellular and extracellular mechanical properties. Front. Oncol., 3 (2013), 145.

[19] Y. Kim, M.A. Stolarska, H.G. Othmer. The role of the microenvironment in tumor growth and invasion. Prog. Biophys. Mol. Biol., 106 (2011), 353-379.

[20] E. Kniazeva, A.J. Putnam. Endothelial cell traction and ECM density influence both capillary morphogenesis and maintenance in 3-D. Am. J. Physiol. Cell Physiol., 297 (2009), C179-C187.

[21] L.D. Landau M. Lifshitz. Theory of Elasticity. London: Pergamon, 1959.

[22] J.R. Lange, B. Fabry. Cell and tissue mechanics in cell migration. Exp. Cell Res., 319 (2013), 2418-2423.

[23] D. Manoussaki, S.R. Lubkin, R.B. Vernon, J.D. Murray. A mechanical model for the formation of vascular networks in vitro. Acta Biotheor., 44 (1996), 271-282.

[24] R.M.H. Merks, S.V. Brodsky, M.S. Goligorsky, S.A. Newman, J.A. Glazier. Cell elongation is key to in silico replication of in vitro vasculogenesis and subsequent remodeling. Dev. Biol., 289 (2006), 44-54.

[25] F. Milde, M. Bergdorf, P. Koumoutsakos. A hybrid model for three-dimensional simulations of sprouting angiogenesis. Biophys. J., 95 (2008), 3146-3160.

[26] J.D. Murray. On the mechanical theory of biological pattern formation with application to vasculogenesis. C.R. Biologies, 326 (2003), 239-252.

[27] P. Namy, J. Ohayon, P. Tracqui. Critical conditions for pattern formation and in vitro tubulogenesis driven by cellular traction fields. J. theor. Biol., 227 (2004), 103-120.

[28] L. Narunsky, R. Oren, F. Bochner, M. Neeman. Imaging aspects of the tumor stroma with therapeutics implications., Pharm. Therap., 141 (2014), 192-208.

[29] Z.K. Otrock, R.A.R. Mahfouz, J.A. Makarem, A.I. Shamseddine. Understanding the biology of angiogenesis: review of the most important molecular mechanisms. Blood Cells Mol. Dis., 39 (2007), 212-220.

[30] M.Z. Pindera, H. Ding, Z. Chen. Convected element method for simulation of angiogenesis. J. Math. Biol., 57 (2008), 467-495.

[31] M.J. Plank, B.D. Sleeman, P.F. Jones. A mathematical model of tumour angiogenesis, regulated by vascular endothelial growth factor and the angiopoietins. J. theor. Biol. 229 (2004), 435-454.

[32] P. Roca-Cusachs, R. Sunyer, X. Trepat. Mechanical guidance of cell migration: lessons from chemotaxis. Curr. Opin. Cell Biol., 25 (2013), 543-549.

[33] D.K. Schlüter, I. Ramis-Conde, M.A.J. Chaplain. Computational modeling of single-cell migration: the leading role of extracellular matrix fibers. Biophys. J., 103 (2012), 1141-1151.

[34] M. Scianna, L. Munaron, L. Preziosi. A multiscale hybrid approach for vasculogenesis and related potential blocking therapies. Prog. Biophys. Mol. Biol., 106 (2011), 450-62.

[35] M. Scianna, C.G. Bell, L. Preziosi. A review of mathematical models for the formation of vascular networks. J. theor. Biol., 333 (2013), 174-209.

[36] D.W. Siemann. The unique characteristics of tumor vasculature and preclinical evidence for its selective disruption by tumor-vascular disrupting agents. Cancer Treat. Rev. 37 (2011), 63-74.

[37] F. Spill, P. Guerrero, T. Alarcon, P.K. Maini, H.M. Byrne. Mesoscopic and continuum modelling of angiogenesis. J. Math. Biol., (2014).

[38] A. Stéphanou, G. Meskaoui, B. Vailhé, P. Tracqui. The rigidity in fibrin gels as a contributing factor to the dynamics of in vitro vascular cord formation. Microvasc. Res. 73 (2007), 182-190.

[39] A. Stéphanou, S.R. McDougall, A.R.A. Anderson, M.A.J. Chaplain. Mathematical modelling of the influence of blood rheological properties upon adaptative tumour-induced angiogenesis. Math. Comp. Mod., 44 (2006), 96-123.

[40] A. Tosin, D. Ambrosi, L. Preziosi. Mechanics and Chemotaxis in the Morphogenesis of Vascular Networks. Bull. Math. Biol., 68 (2006), 1819-1836.

[41] C. Valero, E. Javierre, J.M. García-Aznar, M.J. Gómez-Benito. Numerical modelling of the angiogenesis process in wound contraction. Biochem. Model. Mechanobiol., 12 (2013), 349-360.

[42] M. van Dijk, S.A. Göransson, S. Strömblad. Cell to extracellular matrix interactions and their reciprocal nature in cancer. Exp. Cell Res., 319 (2013), 1663-1670.

[43] R.S. Varga. Matrix iterative analysis. Second ed. (of 1962 Prentice Hall edition), Springer-Verlag, (2002). 